\title{
Hospital-acquired pneumonia: coverage and treatment adequacy of current guidelines
}

\author{
M. Ioanas*, M. Cavalcanti ${ }^{\#}$, M. Ferrer", M. Valencia\#, C. Agusti\#, J. Puig de la Bellacasa ${ }^{\circledR}$, A. Torres ${ }^{\#}$
}

Hospital-acquired pneumonia: coverage and treatment adequacy of current guidelines. M. Ioanas, M. Cavalcanti, M. Ferrer, M. Valencia, C. Agusti, J. Puig de la Bellacasa, A. Torres. (C) ERS Journals Ltd 2003.

ABSTRACT: The American Thoracic Society (ATS) guideline for hospital-acquired pneumonia (HAP) released in 1996 and the Trouillet classification published in 1998 supply different rational foundations for the classification of patients with HAP and for the selection of initial antibiotic therapy. The aims of this study were to assess the level of bacterial coverage and to assess and validate the adequacy of antibiotic strategy of each of these classifications.

Intensive care unit-admitted patients $(n=71)$ with suspicion of HAP were evaluated. The ATS and Trouillet classifications demonstrated an accuracy to predict the causative microorganism of $91 \%$ and $83 \%$, respectively. The ATS and Trouillet antibiotic treatment recommendations were adequate in $79 \%$ and $80 \%$ of the patients, respectively. The microorganisms implicated in the treatment inadequacy of the ATS guideline were Pseudomonas aeruginosa $(n=3)$, Acinetobacter baumanii $(n=1)$, Stenotrophomonas maltophilia $(\mathrm{n}=1)$ and methicillin-resistant Staphylococcus aureus $(n=1)$. $P$. aeruginosa was implicated with Trouillet treatment inadequacy.

The current recommendations for empirical antibiotic treatment of hospital-acquired pneumonia (American Thoracic Society and Trouillet) showed a good ability to predict the involved pathogen. However, considering the resistance pattern of the isolated pathogens, both classifications demonstrated a rather lower treatment adequacy; the main reason was the failure to treat highly resistant strains.

Eur Respir J 2003; 22: 876-882.
*National Institute of Pulmonology "Marius Nasta", Bucharest, Romania. ${ }^{\#}$ Clinic Institute of Pulmonology and Thoracic Surgery, and Dept of Microbiology, Hospital Clinic, Barcelona, Spain.

Correspondence: A. Torres, Institut Clinic de Pneumologia i Cirurgia Toracica, Hospital Clinic, Villarroel 170, 08036 Barcelona, Spain. Fax: 34932279813

E-mail: atorres@medicina.ub.es

Keywords: Guidelines

hospital-acquired pneumonia

treatment adequacy

Received: April 242003

Accepted after revision: June 242003

M. Ioanas was a recipient of a European Respiratory Society Research Fellowship in 2000, M. Cavalcanti in 2003 and M. Valencia in 2002. The study was supported by Red Gira and Red Respira.
The American Thoracic Society (ATS) statement for the management of hospital-acquired pneumonia (HAP) released in 1996, appeared to be extremely well-documented material and a highly useful tool for the treatment of HAP [1]. In 1998, TROUILLET et al. [2] also proposed a classification for patients with ventilator-associated pneumonia (VAP) based on two additional risk factors in order to support another rational basis for initial antibiotic therapy selection.

Despite the creation of these recommendations, studies have confirmed a prominent rate of inappropriate initial treatment [3], which could occur as a result of the presence of an unexpected pathogen or the isolation of a resistant strain of an expected microorganism. In fact, the increasing emergence of bacterial resistance over the last decade could result in a higher level of inadequacy of these initial recommendations for antibiotic treatment. Moreover, the microbial pattern may vary according to different countries and settings, as a result of different strategies for preventing microbial resistance or occurrence of nosocomial infection [4], and this should also be taken into account during the process of choosing the initial therapy.

The mortality rate of HAP remains high at $\sim 30 \%$ [5]. Higher mortality rates are reported in pneumonia caused by Pseudomonas aeruginosa [6] and among patients that receive initial inappropriate antibiotic treatment [3]. The choice of an adequate antimicrobial therapy clearly reduces the hospital stay and further complication [7], and has favourable consequences on the microbial ecology.
The capacity of the ATS guidelines and the Trouillet classification to predict resistant pathogens in patients with HAP and the appropriateness of different antimicrobial regimens in those patients was evaluated by LEROY et al. [8] in 2002, but retrospectively and not taking a clinical outcome into account.

The present study prospectively assessed the level of bacterial coverage of the ATS guidelines and Trouillet classification for HAP and VAP, respectively. Assuming that the changes in the profile of the microbial resistance could alter the adequacy of any therapeutic approach, the adequacy of both ATS and Trouillet antibiotic strategies for nosocomial pneumonia was also assessed and validated.

\section{Methods}

A prospective study was conducted during a 12-month period in the intensive care unit (ICU) of the Hospital Clinic, Barcelona, Spain. The study was approved by the local Ethical Committee.

\section{Patients}

All consecutive patients admitted to the ICU, hospitalised for $>48 \mathrm{~h}$ and with suspicion of HAP were included. The diagnosis of pneumonia was based on the detection of a 
new, persistent pulmonary infiltrate and at least two of the following clinical criteria: 1) fever or hypothermia (temperature $>38^{\circ}$ or $<35.5^{\circ}$ ); 2) leukopaenia or leukocytosis (white blood cells $\leqslant 4 \times 10^{9} \cdot \mathrm{L}^{-1}$ or $\geqslant 12 \times 10^{9} \cdot \mathrm{L}^{-1}$ ); or 3 ) purulent respiratory secretions [9]. Pneumonia was considered ventilatorassociated when it occurred after intubation and was judged not to have been incubated previously. Patients with severe immune suppression (solid organ or bone marrow transplant, human immunodeficiency virus infection, severe neutropaenia, immunosuppressive treatment) were excluded. Patients were followed until hospital discharge or death. Only the first episode of HAP was evaluated.

During the study period, 75 patients with suspicion of HAP were initially included. Four patients were excluded within the following $48 \mathrm{~h}$ because of evidence of an alternative aetiology or complete radiological resolution. In this study, 71 patients were included. The diagnosis of pneumonia was confirmed by the isolation of a potentially pathogenic microorganism or, among those without a defined aetiology, by one of the following: 1) classical clinical presentation associated with adequate response after the beginning of antibiotics; 2) histological confirmation of pneumonia; or 3) exclusion of additional diagnosis. Initial antibiotic treatment was chosen according to the attending physician, without influence of the investigators.

\section{Diagnostic procedures}

Respiratory, blood and urine samples were obtained from the 71 patients before initiating antimicrobial treatment whenever possible. Respiratory samples were obtained by endotracheal aspirate (ETA), protected specimen brush (PSB) and bronchoalveolar lavage (BAL). Collected specimens were processed according to procedures described previously [10]. Respiratory samples were considered valid based on criteria described elsewhere [7]. The significant thresholds defining infection were PSB, BAL and ETA cultures yielding $\geqslant 10^{3}$, $\geqslant 10^{4}$ and $\geqslant 10^{5}$ colony-forming units $\cdot \mathrm{mL}^{-1}$, respectively. Blood cultures, urinary Legionella pneumophila antigen test and detection of Aspergillus sp. antigen in serum was also performed. Thoracentesis with pleural fluid analysis was performed when applicable.

\section{Definition of groups of patients}

Based on initial characteristics each patient was categorised according to the ATS classification [1]. Those patients with VAP were also categorised according to the classification of TROUILLET et al. [2].

The ATS guideline classifies patients into three different groups based on time of onset (before or after 5 days of hospitalisation), severity criteria and the presence of risk factors. Group 1 included patients with mild-to-moderate pneumonia, without risk factors with onset at any time, or severe pneumonia without risk factors with early onset. Group 2 included those with mild-to-moderate pneumonia with risk factors with onset at anytime. Group 3 included those with severe pneumonia without risk factors with late onset, or severe pneumonia with risk factors with onset at any time.

The Trouillet classification stratifies patients with VAP based on the prior duration of mechanical ventilation and the prior use of antibiotic in the previous 15 days. Groups 1 and 2 included patients ventilated for $<7$ days. Group 1 patients had not received prior antibiotic therapy within the last month while group two patients had. Groups 3 and 4 included patients ventilated for $\geqslant 7$ days. Group 3 patients had not received prior antibiotic therapy within the last month while group 4 patients had.

\section{Definitions}

Bacterial coverage. Isolated pathogens from each patient were compared with the predicted spectrum of microorganism according to each patient's category in the ATS and Trouillet classifications. When the isolated pathogen corresponded to the expected one, the bacterial coverage of the ATS or Trouillet classification was considered adequate.

Guideline adherence. Patients were also aggregated into different groups depending on whether the initial empirical treatment chosen by the attending physicians suited the antibiotic treatment suggested by the previously mentioned guidelines or not. Those groups were ATS or non-ATS groups and Trouillet or non-Trouillet groups.

Treatment adequacy. Empirical treatment was considered adequate when the isolated pathogens were susceptible in vitro to at least one of the antibiotics administrated. In case of infection by $P$. aeruginosa, adequate treatment had to consist of at least two active drugs against the isolated strain.

Treatment failure. Treatment was considered to have failed if after $72 \mathrm{~h}$ following initiation, the presence of at least one of the following was defined: 1) persistence of fever $\left(\geqslant 38^{\circ} \mathrm{C}\right)$ or hypothermia $\left(<35.5^{\circ} \mathrm{C}\right)$ plus purulent respiratory secretions; 2$)$ worsening of the pulmonary infiltrates with or without worsening in oxygenation; 3) occurrence of septic shock or multiple organ dysfunction syndrome not present on the first day; or 4) death attributable to pneumonia [7].

\section{Data collection and end-points}

Demographic and clinical parameters included the Acute Physiology and Chronic Health Evaluation II [11], time of pneumonia onset, severity criteria and risk factors [1], microbiological results, antimicrobial treatment for nosocomial pneumonia, lengths of mechanical ventilation, ICU and hospital stay.

The end-points analysed were adequate bacterial coverage, treatment adequacy, treatment failure, hospital mortality and lengths of ICU and hospital stay. Hospital mortality was defined as death by any cause that occurred within hospitalisation.

\section{Statistical analysis}

Results are expressed as percentage and mean \pm SD. The continuous variables were compared using the Mann-Whitney U-test and categorical variables were contrasted by the Chisquared test or Fisher's exact test. The level of significance was set at $5 \%$.

\section{Results}

Seventy-one patients with HAP were evaluated. Epidemiological characteristics at the time of ICU admission are detailed in table 1. Of these patients, $29(40.8 \%)$ cases of HAP were ventilator-associated. The mean duration of mechanical ventilation before VAP onset was $5.4 \pm 2.7$ days (range 1-11 days). Nineteen $(26.8 \%)$ patients required intubation after 
Table 1. - Intensive care unit (ICU) admission characteristics of 71 patients studied prospectively

\begin{tabular}{lc}
\hline Characteristic & Value \\
\hline Age yrs & $62.7 \pm 13.9$ \\
Male & $55(77.5)$ \\
APACHE II & $14.9 \pm 5.6$ \\
Comorbid conditions & \\
Coma & $10(14.1)$ \\
Head trauma & $5(7.0)$ \\
Renal failure & $3(4.2)$ \\
Diabetes mellitus & $7(9.9)$ \\
Underlying lung disease & $23(32.4)$ \\
Recent surgery & $38(53.5)$ \\
Aspiration & $11(15.5)$ \\
Antibiotics during previous month & $50(70.4)$ \\
Cause of ICU admission & \\
Postoperative & $38(53.5)$ \\
Exacerbated COPD & $4(5.6)$ \\
Pneumonia & $3(4.2)$ \\
Neurological & $5(7.0)$ \\
Trauma & $5(7.0)$ \\
Other pulmonary disease & $4(5.6)$ \\
Miscellaneous & $12(16.9)$ \\
Severity criteria & $57(80.3)$ \\
Definitive aetiology & $33(46.5)$ \\
VAP & $29(40.8)$ \\
\hline
\end{tabular}

Data are presented as mean \pm SD or $\mathrm{n}(\%)$ unless otherwise stated. APACHE: Acute Physiology and Chronic Health Evaluation; COPD: chronic obstructive pulmonary disease; VAP: ventilator-associated pneumonia.

onset of HAP, and in this group the mean time of mechanical ventilation was a $9.0 \pm 3.8$ days.

\section{Microbiological findings}

An aetiological diagnosis was achieved in 33 patients $(46.5 \%)$ and a total number of 48 pathogens were isolated. Polymicrobial pneumonia was diagnosed in 14 patients $(19.7 \%)$. The most frequently isolated microorganisms were P. aeruginosa $(\mathrm{n}=9,18 \%)$ and methicillin-resistant Staphylococcus aureus (MRSA; $n=8,16 \%$ ). Positive blood cultures with agents considered as causative of pneumonia were found in six patients (Klebsiella oxytoca, Enterobacter aerogenes, $P$. aeruginosa, Escherichia coli, Enterobacter cloacae and MRSA). Aetiological diagnosis of pleural empyema was achieved in three of five cases, and the causative agents were MRSA in two cases and coinfection of Streptococcus milleri and $E$. coli in another case. Four patients with negative serum Aspergillus antigen had Aspergillus fumigatus isolated in association with a Gram-negative strain and were not considered pathogenic. One patient submitted to surgical resection of lung aspergiloma developed a postoperative pneumonia with no other microorganism isolated besides Aspergillus, had a positive serum Aspergillus antigen, and therefore the fungus was considered the causative agent. Legionella urinary antigen was performed in $49 \%$ of patients, without any positive results.

\section{Group distribution}

The distribution of patients and their pathogens according to the ATS guideline is described in table 2 . No patient complied with ATS group 1 criteria. Of the 29 cases of VAP, $18(62.1 \%)$ had a defined aetiological diagnosis. These
Table 2.-Distribution of pathogens according to American Thoracic Society (ATS) statement

\begin{tabular}{|c|c|c|c|}
\hline & \multirow[t]{2}{*}{ Total } & \multicolumn{2}{|c|}{ ATS guideline } \\
\hline & & Group 2 & Group 3 \\
\hline Total patients & 71 & 14 & 57 \\
\hline Patients with defined aetiology & 33 & 6 & 27 \\
\hline Isolated microorganism & 48 & 9 & 39 \\
\hline Potentially resistant & 20 & 4 & 16 \\
\hline Pseudomonas aeruginosa & 9 & 3 & 6 \\
\hline Acinetobacter sp. & 1 & & 1 \\
\hline Stenotrophomonas maltophilia & 2 & & 2 \\
\hline MRSA & 8 & 1 & 7 \\
\hline Other & 28 & 5 & 23 \\
\hline Klebsiella sp. & 1 & & 1 \\
\hline Escherichia coli & 5 & 2 & 3 \\
\hline Enterobacter $\mathrm{sp}$. & 1 & & 1 \\
\hline Serratia sp. & 1 & & 1 \\
\hline Proteus sp. & 1 & & 1 \\
\hline Citrobacter sp. & 2 & & 2 \\
\hline Haemophilus influenzae & 4 & 1 & 3 \\
\hline Streptococcus pneumoniae & 2 & 1 & 1 \\
\hline Moraxella catarrhalis & 1 & & 1 \\
\hline MSSA & 4 & & 4 \\
\hline Aspergillus & 5 & & 5 \\
\hline Streptococcus milleri & 1 & 1 & \\
\hline
\end{tabular}

Data are presented as n. MRSA: methicillin-resistant Staphylococcus aureus; MSSA: methicillin-sensible Staphylococcus aureus.

patients were also classified according to the Trouillet classification and their distribution is shown in table 3 .

The empirical therapy chosen by the attending physician fulfilled the ATS treatment recommendation in $52(73.2 \%)$ patients, who were designated ATS group. Conversely, those $19(26.8 \%)$ who were not treated according to the ATS recommendation were designated non-ATS group. Only eight of the $29(27.6 \%)$ patients with VAP received an initial antibiotic therapy that followed the Trouillet suggested treatment (Trouillet group), whereas 21 patients received a different sort of treatment (non-Trouillet group). All the different antibiotic combinations employed are shown in table 4 .

\section{Bacterial coverage}

The ATS classification could correctly predict the isolated microorganisms in 30 of $33(90.9 \%)$ patients. It failed to predict the pathogens isolated from two patients classified in the ATS group 2 ( $P$. aeruginosa and MRSA) and one pathogen from an ATS group 3 patient (Aspergillus). The specific risk factors of each pathogen were $P$. aeruginosa (use of corticoids), MRSA (postoperative lung cancer resection, alcohol ingestion and chronic obstructive pulmonary disease (COPD)), Aspergillus (postoperative pulmonary aspergiloma resection).

The use of the Trouillet classification could properly predict the isolated microorganism in 15 of $18(83.3 \%)$ patients, only failing to predict three pathogens: one MRSA in a Trouillet group 1 patient (postoperative lung cancer resection, alcohol ingestion), one MRSA in a Trouillet group 2 patient (previous hospitalisation and previous use of antibiotics), and an Aspergillus in a Trouillet group 4 patient (postoperative pulmonary aspergiloma resection).

\section{Treatment adequacy}

The ATS antibiotic treatment strategy was adequate in 19 of $24(79.1 \%)$ patients that had a defined aetiological diagnosis. 
Table 3.-Distribution of pathogens according to Trouillet classification

\begin{tabular}{|c|c|c|c|c|c|}
\hline & \multirow[t]{2}{*}{ Total } & \multicolumn{4}{|c|}{ Trouillet classification } \\
\hline & & Group 1 & Group 2 & Group 3 & Group 4 \\
\hline Total patients & 29 & 6 & 11 & 2 & 10 \\
\hline Patients with defined aetiology & 18 & 3 & 6 & 1 & 8 \\
\hline Isolated microorganism & 25 & 5 & 8 & 1 & 11 \\
\hline Potentially resistant & 8 & 1 & 3 & & 4 \\
\hline Pseudomonas aeruginosa & 3 & & 2 & & 1 \\
\hline Acinetobacter sp. & 1 & & & & 1 \\
\hline Stenotrophomonas maltophilia & 1 & & & & 1 \\
\hline MRSA & 3 & 1 & 1 & & 1 \\
\hline Other & 17 & 4 & 5 & 1 & 7 \\
\hline \multicolumn{6}{|l|}{ Klebsiella sp. } \\
\hline Escherichia coli & 2 & 1 & 1 & & \\
\hline Enterobacter sp. & 1 & 1 & & & \\
\hline Serratia sp. & 1 & & & & 1 \\
\hline Proteus sp. & 1 & & & & 1 \\
\hline Citrobacter sp. & 2 & & 1 & & 1 \\
\hline Haemophilus influenzae & 3 & 1 & 1 & & 1 \\
\hline \multicolumn{6}{|l|}{ Streptococcus pneumoniae } \\
\hline \multicolumn{6}{|l|}{ Moraxella catarrhalis } \\
\hline MSSA & 4 & 1 & 1 & 1 & 1 \\
\hline Aspergillus & 3 & & 1 & & 2 \\
\hline Streptococcus milleri & & & & & \\
\hline
\end{tabular}

Data are presented as n. MRSA: methicillin-resistant Staphylococcus aureus; MSSA: methicillin-sensible Staphylococcus aureus.

Among those patients treated according to the ATS recommendation, treatment inadequacy occurred in six patients, all of them from ATS group 3. The six implicated pathogens were three resistant strains of $P$. aeruginosa (postoperative lung cancer resection, alcohol ingestion, neoplasia, prolonged use of corticoids, previous antibiotics), one of $A$. baumannii (alcohol ingestion, COPD), one of Stenotrophomonas maltophilia (alcohol ingestion, prolonged use of corticoids, previous antibiotics) and one MRSA (postoperative lung resection, alcohol ingestion).

The Trouillet treatment recommendation was adequate in four of five $(80 \%)$ patients treated according to this classification and with an aetiological diagnosis defined. Treatment inadequacy among those treated as suggested by this classification occurred in only one patient from Trouillet group 2, who presented with a resistant strain of $P$. aeruginosa, and had risk factors of postoperative lung cancer resection, alcohol ingestion, neoplasia, prolonged use of corticoids, and previous antibiotic use.

\section{Outcomes}

There was a tendency toward higher treatment adequacy in the ATS compared with the non-ATS group $(79.2 \%$ and $55.6 \%$ ), although significance was not achieved. More patients from the ATS group presented treatment failure $(66 \%)$, but there was no difference in the overall mortality between the ATS and non-ATS groups. Moreover, no difference in the length of ICU and hospital stay could be demonstrated among patients from both groups. The influence of the adherence to the ATS treatment recommendation on these end-points is detailed in table 5 .

The empirical therapy of the Trouillet group also demonstrated a tendency toward superior treatment adequacy compared with the non-Trouillet group (80\% and $69.2 \%)$, but without significance. Treatment failure occurred in $62.5 \%$ of the Trouillet and $52.4 \%$ in the non-Trouillet group. No difference in ICU or hospital stay was found between the Trouillet and non-Trouillet groups. There was a $50 \%$ mortality in the Trouillet and a $38 \%$ in the non-Trouillet groups; however, significance was not achieved. These results are shown in table 6 .

\section{Discussion}

This study found that the current ATS guideline for the management of HAP has a high accuracy to predict the causative microorganism $(91 \%)$. In this setting, and taking into account in vitro susceptibility of the pathogen, the adequacy of the ATS treatment was rather lower $(79 \%)$. The ATS guideline mainly failed to cover highly resistant organisms such as $P$. aeruginosa, $S$. maltophilia and MRSA. The Trouillet classification demonstrates an $83 \%$ accuracy to predict the causal pathogen. Again, when considering the bacterial resistance profile, treatment recommendations resulted in an $80 \%$ adequacy. The Trouillet classification only failed to cover a resistant strain of $P$. aeruginosa. No differences were found in mortality between patients that were treated, or not, according to both recommendations.

The association between mortality of HAP and inappropriate antibiotic therapy has been intensely investigated over the last years. Although some studies [3,12] found no significant differences, others showed a significantly higher mortality among those patients that received inadequate initial treatment $[6,13]$ or when there was a delay in initiating treatment [14]. Furthermore, there is a general agreement that inadequate treatment is related to the emergence of resistant pathogens [15, 16] and to a prolonged ICU stay [17]. Inadequacy of the empirical treatment can occur as a result of the presence of an unexpected microorganism or the isolation of a resistant strain of an expected pathogen. The guidelines are developed to predict microbial aetiology and to help clinicians in prescribing initial empirical adequate therapy. Consequently, the clinical validation of guidelines in prospective studies is very important.

Two studies had previously evaluated the adequacy of the ATS and Trouillet classifications regarding the prediction of pathogens, with controversial results. RELLO et al. [18] found 
Table 4.-Isolated microorganisms and empirical antibiotic treatment

\begin{tabular}{|c|c|c|c|c|c|c|c|}
\hline & \multirow{2}{*}{\multicolumn{2}{|c|}{ Group }} & \multirow{4}{*}{ Microorganisms } & \multirow{4}{*}{ Empirical treatment } \\
\hline \multirow{2}{*}{\multicolumn{2}{|c|}{ Group }} & \multirow{4}{*}{ Microorganisms } & \multirow{4}{*}{ Empirical treatment } & & & & \\
\hline & & & & \multirow{2}{*}{ ATS } & \multirow{2}{*}{ Trouillet } & & \\
\hline ATS & Trouillet & & & & & & \\
\hline & & & & $3^{\#}$ & & No growth & AMI, IMI \\
\hline $3^{\#}$ & $4^{\#}$ & MRSA & IMI, CIP, VAN & $3^{\#}$ & & No growth & CIP, IMI, FLU \\
\hline \multirow[t]{2}{*}{$3^{\#}$} & \multirow[t]{5}{*}{4} & P. aeruginosa, & CEFT, CIP & $3^{\#}$ & & No growth & CIP, IMI \\
\hline & & Acinotobacter sp. & & $3^{\#}$ & & No growth & AMI, CEFT, VAN \\
\hline 3 & & MRSA & CEF, AMI, CLA & $3^{\#}$ & 2 & No growth & CIP, PIP-TAZ, VAN \\
\hline \multirow[t]{2}{*}{$2^{\#}$} & & No growth & AMI, PIP-TAZ, & 3 & & No growth & IMI, VAN \\
\hline & & & VAN & $3^{\#}$ & $2^{\#}$ & MSSA & PIP-TAZ, CIP \\
\hline $3^{\#}$ & \multirow{5}{*}{$\begin{array}{l}2 \\
2^{\#}\end{array}$} & Citrobacter sp. & IMI, AMI & & & H. influenzae & \\
\hline $3^{\#}$ & & P. aeruginosa & CEFT, CIP & $3^{\#}$ & & P. aeruginosa, & CEF, AMI \\
\hline \multirow[t]{3}{*}{$3^{\#}$} & & E. coli, Aspergillus, & VAN, AMI, & & & Aspergillus & \\
\hline & & MRSA & PIP-TAZ, AMP B, & 3 & $1^{\#}$ & No growth & AMOX-CLAV \\
\hline & & & TMP-SMZ & 3 & & No growth & CTX, CLIN \\
\hline $3^{\#}$ & \multirow[t]{3}{*}{3} & No growth & IMI, AMI & $3^{\#}$ & & No growth & CEF, AMI \\
\hline \multirow[t]{2}{*}{$3^{\#}$} & & S. pneumoniae, & $\mathrm{CEF}, \mathrm{CIP}$ & $3^{\#}$ & 1 & No growth & CIP, CEFT \\
\hline & & M. catarralis & & $3^{\#}$ & $2^{\#}$ & No growth & CEF, CIP \\
\hline \multirow[t]{2}{*}{3} & \multirow{4}{*}{1} & No growth & AMP B, CEF, & $3^{\#}$ & 4 & Aspergillus & IMI, CIP, AMP B \\
\hline & & & CLIN, PIP-TAZ & 3 & 1 & E. coli, & IMI, VAN \\
\hline $3^{\#}$ & & No growth & CEF, CIP & & & E. aerogenes & \\
\hline 3 & & No growth & $\mathrm{AZT}, \mathrm{CEF}$ & 3 & $1^{\#}$ & MSSA, & CTX, CLIN \\
\hline $2^{\#}$ & \multirow[t]{6}{*}{4} & No growth & PIP-TAZ, VAN & & & H. influenzae & \\
\hline $2^{\#}$ & & E. coli & CEF, CIP & $2^{\#}$ & & No growth & CTX, ERI \\
\hline $2^{\#}$ & & No growth & IMI, AMI & $2^{\#}$ & & $P$. aeruginosa & IMI, VAN, AMI \\
\hline $3^{\#}$ & & No growth & CIP, PIP-TAZ & 3 & 2 & E. coli, & VAN \\
\hline \multirow[t]{2}{*}{2} & & H. influenzae, & LEV, CLA & & & Aspergillus & \\
\hline & & $\begin{array}{c}\text { S. pneumoniae, } \\
\text { MRSA }\end{array}$ & & $2^{\#}$ & & $\begin{array}{l}\text { E. coli, } \\
\text { S. milleri }\end{array}$ & IMI, VAN, AMI \\
\hline $3^{\#}$ & & No growth & $\begin{array}{c}\text { AMI, CEFT, } \\
\text { FLU, VAN }\end{array}$ & $3^{\#}$ & 4 & $\begin{array}{l}\text { C. freundii } \\
\text { P. mirabilis }\end{array}$ & $\mathrm{CEF}, \mathrm{AMI}$ \\
\hline
\end{tabular}

No growth

$P$. aeruginosa

No growth

MRSA

MRSA

No growth

$P$. aeruginosa

MSSA

No growth

$P$. aeruginosa

No growth

No growth

$H$. influenzae

No growth

MRSA

MRSA,

S. malthophilia

MSSA

K. oxytoca

No growth

No growth

$P$. aeruginosa

No growth

No growth

4

S. marcescens

No growth

No growth

S. maltophilia,

Aspergillus

$P$. aeruginosa

No growth

No growth

No growth

No growth

Table 4. - Continued

GENT, IMI

IP, CEFT

$$
\text { IMI }
$$

FLU, CTX

CEF, AMI

FLU, PIP-TAZ

PIP-TAZ, AMI

CTX, CLIN

AMI, IMI

CEF, AMI

CEFT, CIP

CEF, TEI

CEF, AMI

AMI, IMI

IMI, CIP

QUIN-DALF,

TOB, AZT,

TMP-SMZ

IMI, AMI

IMI, CIP

TMP-SMZ, ERI,

FLU, IMI, TB,

VAN, ACY

CEF, AMI

CEF, CIP

CEFT, CIP

CIP, IMI

CEF, AMI

AMI, CEF

CIP, CEF

LEV, TMP-SMZ

CEF, CIP

AMI, CEF

AMOX-CLAV

CIP, IMI, VAN

CIP, IMI, TEI

MRSA: methicillin-resistant Staphylococcus aureus; IMI: imipenem; CIP: ciprofloxacin; VAN: vancomycin; $P$. aeruginosa: Pseudomonas aeruginosa; CEFT: ceftazidime; CEF: cefepime; AMI: amikacin; CLA: clarithromycin; PIP-TAZ: piperacillin-tazobactam; E. coli: Escherichia coli; TMP-SMZ: trimethoprim-sulphamethoxazole; AMP: amphotericin; S. pneumoniae: Streptococcus pneumoniae; M. catarrhalis: Moraxella catarralis; CLIN: clindamycin; AZT: aztreonam; H. influenzae: Haemophilus influenzae; S. malthophilia: Stenotrophomonas malthophilia; LEV: levofloxacin; FLU: fluconazole; GENT: gentamycin; CTX ceftriaxone; MSSA: methicillin-sensible Staphylococcus aureus; TEI teicoplamin; QUIN-DALF: quinupristin-dalfopristin; TOB: tobramycin; K. oxytoca: Klebsiella oxytoca; ERI: erithromycin; TB: tuberculostatics; ACY: acyclovir; $S$. marcescens: Serratia marcescens; AMOX-CLAV: amoxacillin-clavulanate; $E$. aerogenes: Enterobacter aerogenes; $S$ milleri: Streptococcus milleri; $C$. freundii: Citrobacter freundii;

P. mirabilis: Proteus mirabilis; ${ }^{\#}$ : prescribed antibiotic treatment that suited recommendations.

a significant variation in the aetiology of microorganisms isolated across three different ICUs (Seville and Tarragona, Spain; Montevideo, Uruguay). They found that both the ATS guideline and the Trouillet classification failed to predict the presence of highly resistant pathogens (Pseudomonas) in some patients belonging to low-risk groups. Contrarily, LEROY et al. [8] found a 100\% accuracy of prediction when using the ATS guideline to exclude the presence of resistant pathogens in low-risk patients. In the present study, highly resistant pathogens were found in low-risk patients from the ATS group 2 and the Trouillet groups 1 and 2. The overall rate of prediction was very good for both classifications. Further studies should address the specific risk factors related to the presence of unexpected pathogens in low-risk classes in the ATS guidelines and the Trouillet classification. In this study, most of the unexpected pathogens were isolated in patients that had pulmonary surgery.

One of the problems of the guidelines, when predicting adequate initial antibiotic regimes, is the potential presence of resistance of microorganisms to antibiotics. This issue has 
Table 5.-Outcomes according to adequacy of American Thoracic Society (ATS) treatment recommendation

\begin{tabular}{lrcc}
\hline Outcomes & ATS & Non-ATS & p-value \\
\hline Subjects n & 52 & 19 & \\
Treatment adequacy & $19 / 24(79.2)$ & $5 / 9(55.6)$ & NS \\
Failure of treatment & $33(66.0)$ & $10(47.6)$ & NS \\
Mortality & $17(34.0)$ & $7(33.3)$ & NS \\
LOS hospital & $33.0 \pm 19.4$ & $26.2 \pm 10.8$ & NS \\
LOS ICU & $18.5 \pm 12.4$ & $16.4 \pm 9.7$ & NS \\
\hline
\end{tabular}

Data are presented as $\mathrm{n}(\%)$ or median \pm SD unless otherwise stated. LOS: length of stay; ICU: intensive care unit. NS: nonsignificant. ${ }^{\#}$ : only those with isolated pathogen were included in this analysis.

been covered, for example, in the last ATS guidelines on community-acquired pneumonia with regards to Streptococcus pneumoniae resistant to antibiotics [19]. However, the issue of resistance in HAP is much more complex due to the tremendous variation of the resistance patterns across units and countries. The knowledge of the risk factors for specific resistances is crucial for the administration of adequate empirical antibiotic regimes in HAP and VAP.

Over the last decade, bacterial resistance in ICUs has been a constant challenge for the clinicians. Since 1995, when the ATS statement for the management of HAP was first released [1], bacterial resistance had a considerable spread [4, 20] and several studies focused on the risks and consequences of infection by resistant strains [21, 22]. In this study, 38\% of the isolated strains were resistant and this was the major cause of failure on both strategies of treatment. Basically, three pathogens were involved: $P$. aeruginosa, MRSA and $S$. maltophilia. Concerning $P$. aeruginosa, three of the nine isolated strains showed resistance to both antibiotics used for treatment, resulting in the inadequacy of treatment. As reported in several studies, the leading aetiological organism in HAP appears to be $P$. aeruginosa, isolated in $24 \%$ of cases [23], which is also associated with a higher mortality compared with other pathogens [5]. Local and periodical surveillance studies are strongly recommended to determine the antibiotic patterns of sensitivity of this difficult-to-treat microorganism.

A surprising finding in the present study was that the traditional risk factors related to highly resistant organisms, especially previous use of antibiotics and prolonged ICU stay [2], were not always present. For example, two of eight patients with an early onset pneumonia and MRSA were not at risk for this specific pathogen. Under these circumstances, the likelihood of spread of resistant strains into the community must be considered. A recent study [24] found a number of community infections by MRSA, drawing attention to the dissemination of this pathogen outside the hospital area. The use of vancomycin in the empirical treatment of VAP has been recommended in a very recent study from IBRAHIM et al. [25]. Using this approach the authors achieved a $90 \%$ adequacy in the empirical treatment. De-escalation therapy was used subsequently. Interestingly, antibiotics were stopped after 7 days of treatment. With this strategy, they reduced antibiotic resistance when compared with a control population. A more balanced view of the problem of MRSA, instead of giving vancomycin indiscriminately to all patients with suspicion of VAP, should include precautions of the development of Enterococcus faecium resistant to vancomycin. In this view, again, the authors' recommendation is to develop better models of prediction for the presence of MRSA infection and to include these models in local guidelines.

Infection by $S$. maltophilia had a slightly higher incidence
Table 6.-Outcomes according to adequacy of Trouillet treatment recommendation

\begin{tabular}{lccc}
\hline Outcomes & Trouillet & Non-Trouillet & p-value \\
\hline Subjects n & 8 & 21 & \\
Treatment adequacy & $4 / 5(80)$ & $9 / 13(69.2)$ & NS \\
Treatment failure & $5(62.5)$ & $11(52.4)$ & NS \\
Mortality & $4(50)$ & $8(38.1)$ & NS \\
LOS hospital & $22.7 \pm 11$ & $30.8 \pm 23.7$ & NS \\
LOS UCI & $17.1 \pm 9.5$ & $21.8 \pm 11.6$ & NS \\
\hline
\end{tabular}

Data are presented as $\mathrm{n}(\%)$ or median \pm SD unless otherwise stated. LOS: length of stay; ICU: intensive care unit. NS: nonsignificant. ${ }^{\#}$ : only those with isolated pathogen were included in this analysis.

in this study compared with other reports $(4.1 \%$ compared with $1.7 \%$ in the pooled incidence calculated by CHASTRE and FAGON [23]). At the moment trimethoprim-sulphamethoxazole (TMP-SMZ) is the antibiotic of choice for the treatment of S. maltophilia infections with $>90 \%$ in vitro susceptibility, followed by ticarcillin clavulanate or ceftazidime with $50 \%$ susceptibility [26]. Given the relative lack of agents that have significant activity against $S$. maltophilia, it is not surprising that this pathogen is virtually almost never covered by the Trouillet or ATS treatments. In fact, this microorganism and other nonfermenting Gram-negative bacilli are not mentioned in the lists of the ATS guideline and the Trouillet classification. A recent study [27], reports a number of risk factors for nosocomial pneumonia by $S$. maltophilia in trauma patients (cefepime exposure, tracheostomy, pulmonary contusion and increased morbidity) and suggests the association of an agent with activity against this microorganism (preferably TMPSMZ) when these conditions are present. New guidelines should also cover this problem.

Although $A$. fumigatus was isolated in five patients, only one case was included in the analysis in which it was definitely considered the causative pathogen. Exclusion of the other cases are justified by the following reasons: 1) in most cases the fungus was associated to another organism (E. coli and $S$. maltophilia), which was taken into account for the evaluation of the treatment; 2) no specific serum antigen of $A$. fumigatus was detected; and 3) those patients were immunocompetent and did not have previous use of corticoids, a traditional risk factor associated with fungal infection. However, the ATS guidelines and the Trouillet classification do not deal with the problem of Aspergillus and again this issue should be covered in future guidelines.

No differences in mortality or morbidity were found when comparing patients treated, or not, according to the guidelines, and this is probably due to a relatively small sample size. It is also important to note that there were no patients from the ATS group 1, and these conclusions should not be extended to this group. Other studies in community-acquired pneumonia [28] have found that the application of guidelines (in this case from the ATS, 1993) resulted in a lower mortality. In a recent study, applying a specific treatment protocol for VAP resulted in a lower morbidity [25]. Although the ATS guideline and the Trouillet classification adequately predict the aetiological microorganisms of pneumonia in a high percentage of cases, a potential limitation to generalise these results is the variability of the local antimicrobial resistance profiles among different ICUs that may decrease the clinical efficacy of these recommendations.

To conclude, the current classifications for empirical antibiotic treatment of hospital-acquired pneumonia (American Thoracic Society and Trouillet) showed a good ability to predict the involved pathogen. However, considering the resistance pattern of isolated pathogens, both classifications 
demonstrated a rather lower treatment adequacy; the main reason was the failure to treat highly resistant strains. Additional parameters, such as local microbial epidemiology and more accurate models of prediction of resistance, should be considered in order to improve the level of coverage and adequacy of the antibiotic treatment. Future guidelines should address the role of other microorganisms such as Stenotrophomonas maltophilia and Aspergillus sp.

\section{References}

1. American Thoracic Society. Hospital acquired-pneumonia in adults: diagnosis assessment, initial therapy and prevention a consensus statement. Am J Respir Crit Care Med 1996; 153: $1711-1725$.

2. Trouillet JL, Chastre J, Vuagnat A, et al. Ventilatorassociated pneumonia caused by potentially drug-resistant bacteria. Am J Respir Crit Care Med 1998; 157: 531-539.

3. Alvarez-Lerma F, and the ICU Study Group. Modification of the empiric treatment in patients with pneumonia acquired in the intensive care unit. Intensive Care Med 1996; 22: 387-394.

4. Kollef MH, Fraser VJ. Antibiotic resistance in intensive care unit. Ann Intern Med 2001; 134: 298-314.

5. Crouch Brewer S, Jones CB, Leeper KV, Wunderink RG. Ventilator-associated pneumonia due to Pseudomonas aeruginosa. Chest 1996; 109: 1029.

6. Luna CM, Vujacich P, Niederman MS, et al. Impact of BAL data on the therapy and outcome of ventilator-associated pneumonia. Chest 1997; 111: 685.

7. Ioanas M, Ferrer R, Angrill A, Ferrer M, Torres A. Microbial investigation in ventilator-associated pneumonia. Eur Respir J 2001; 17: 791-801.

8. Leroy O, Giradie P, Yazdanpanah Y, et al. Hospitalacquired pneumonia: microbiological data and potential adequacy of antimicrobial regimens. Eur Respir $J$ 2002; 20: 432-439.

9. Johanson WG Jr, Pierce AK, Sanford JP, Thomas GD. Nosocomial respiratory infections with Gram-negative bacilli: the significance of colonization of the respiratory tract. Ann Intern Med 1972; 77: 701-706.

10. Ruiz M, Torres A, Ewig S, et al. Noninvasive versus invasive microbial investigation in ventilator-associated pneumonia. Evaluation of outcome. Am J Respir Crit Care Med 2000; 162: 119-125.

11. Knaus WA, Zimmerman JE, Wagner DP, Draper EA, Lawrence DE. APACHE: acute physiology and chronic health evaluation - a physiologically based classification system. Crit Care Med 1981; 9: 591-597.

12. Sanchez-Nieto AM, Torres A, Garcia-Cordoba A, et al. Impact of invasive and non-invasive quantitative culture sampling of ventilator-associated pneumonia. Am $J$ Respir Crit Care Med 1998; 157: 371-376.

13. Kollef MH, Sherman G, Ward S, Fraser VJ. Inadequate antimicrobial treatment of infections. A risk factor for hospital mortality among critically ill patients. Chest 1999; 115: $462-474$.
14. Iregui M, Ward S, Sherman G, Fraser V, Kollef M. Clinical importance of delays in the initiation of appropriate antibiotic treatment for ventilator-associated pneumonia. Chest 2002; 122: 262-268.

15. Grusson D, Hilbert G, Vargas F, et al. Rotation and restricted use of antibiotics in a medical intensive care unit. Am J Respir Crit Care Med 2000; 162: 837-843.

16. Kollef $\mathrm{MH}$, Ward $\mathrm{S}$, Sherman G. Inadequate treatment of nosocomial infections is associated with certain empiric antibiotic choices. Crit Care Med 2000; 28: 3456-3464.

17. Dupont H, Mentec H, Sollet JP, Bleichner G. Impact of appropriateness of initial antibiotic therapy on the outcome of ventilator-associated pneumonia. Intensive Care Med 2001; 27: 355-362.

18. Rello J, Sa-Borges M, Correa H, Leal SR, Baraibar J. Variations in etiology of ventilator-associated pneumonia across four treatment sites. Am J Respir Crit Care Med 1999; 160: 608-613.

19. American Thoracic Society. Guidelines for the management of adults with community-acquired pneumonia. Diagnosis, assessment of severity, antimicrobial therapy and prevention. Am J Respir Crit Care Med 2001; 163: 1730-1754.

20. National Nosocomial Infections Surveillance (NNIS) System. National Nosocomial Infections Surveillance System report, data summary from January 1990-May 1999, issued June 1999. Am J Infect Control 1999; 27: 520-532.

21. Fagon JY, Chastre J, Comart Y, Trouillet JL, Gilbert C. Mortality due to ventilator-associated pneumonia or colonization with Pseudomonas or Acinetobacter species: assessment by quantitative culture of samples obtained by a protected specimen brush. Clin Infect Dis 1996; 23: 538542 .

22. Hanberger H, Garcia-Rodriguez JA, Bobernado M, Goossens $\mathrm{H}$, Nilsson LE, Struelens MJ. Antibiotic susceptibility among aerobic gram-negative bacilli in intensive care units in 5 European countries. French and Portuguese ICU Study Groups. JAMA 1999; 281: 67-71.

23. Chastre J, Fagon JY. Ventilator-associated pneumonia. Am J Respir Crit Care Med 2002; 165: 867-902.

24. Naimi TS, LeDell KH, Boxrud DJ, Groom AV, Steward CD. Epidemiology and clonality of community-acquired MRSA in Minnesota, 1996-1998. Clin Infect Dis 2001; 33: 990-996.

25. Ibrahim EH, Ward S, Sherman G, Schaiff R, Fraser VJ, Kollef MH. Experience with a clinical guideline for the treatment of ventilator-associated pneumonia. Crit Care Med 2001; 29: 1109-1115.

26. Betriu C, Sanchez A, Palau ML, Gomez M, Picazo IL. Antibiotic resistance surveillance of Stenotrophomonas maltophilia. 1993-1999. J Antimicrob Chemother 2001; 48: 152-154.

27. Hanes SD, Demirkan K, Tolley E, et al. Risk factors for lateonset nosocomial pneumonia caused by Stenotrophomonas maltophilia in critically ill trauma patients. Clin Infect Dis 2002; 35: 228-235.

28. Dean NC, Silver MP, Bateman KA, James B, Hadlock CJ, Hale D. Decrease mortality after implementation of a treatment guideline for community-acquired pneumonia. Am J Med 2001; 110: 451-457. 